Proceedings of the

International Geometry Center

Vol. 10, no. 2 (2017) pp. 8-23

\title{
Warped product semi-slant submanifolds in locally conformal Kaehler manifolds
}

\author{
Koji Matsumoto
}

\begin{abstract}
In 1994, in [13], N. Papaghiuc introduced the notion of semi-slant submanifold in a Hermitian manifold which is a generalization of $C R$ - and slant-submanifolds. In particular, he considered this submanifold in Kaehlerian manifolds, [13]. Then, in 2007, V. A. Khan and M. A. Khan considered this submanifold in a nearly Kaehler manifold and obtained interesting results, [11]. Recently, we considered semi-slant submanifolds in a locally conformal Kaehler manifold and gave a necessary and sufficient conditions for two distributions (holomorphic and slant) to be integrable. Moreover, we considered these submanifolds in a locally conformal Kaehler space form, [4]. In this paper, we define 2-kind warped product semi-slant submanifolds in a locally conformal Kaehler manifold and consider some properties of these submanifolds.
\end{abstract}

\section{INTRODUCTION}

A Hermitian manifold $\widetilde{M}$ with structure $(J, \widetilde{g})$ is called a locally conformal Kaehler (an l.c.K.-) manifold if each point $x \in \widetilde{M}$ has an open neighbourhood $U$ with differentiable function $\rho: U \rightarrow \mathcal{R}$ such that $\widetilde{g}^{*}=e^{-2 \rho} \widetilde{g}_{\mid U}$ is a Kaehlerian metric on $U$, that is, $\nabla^{*} J=0$, where $J$ is the almost complex structure, $\widetilde{g}$ is the Hermitian metric, $\nabla^{*}$ is the covariant differentiation with respect to $\widetilde{g}^{*}$ and $\mathcal{R}$ is a real number space, [14]. A typical example of an l.c.K.-manifold which is not Kaehlerian is Hopf manifold, [14].

Then we know the following statement, see [10]:

2010 Mathematics Subject Classification: 53C40

Keywords: Locally conformal Kaehler manifold, slant distribution, semi-slant submanifold, warped product semi-slant submanifold 
Proposition 1.1. A Hermitian manifold $\widetilde{M}(J, \widetilde{g})$ is l.c.K. if and only if there exists a global closed 1-form $\alpha$ which is called Lee form satisfying

$$
\left(\widetilde{\nabla}_{V} J\right) U=-\widetilde{g}\left(\alpha^{\sharp}, U\right) J V+\widetilde{g}(V, U) \beta^{\sharp}+\widetilde{g}(J V, U) \alpha^{\sharp}-\widetilde{g}\left(\beta^{\sharp}, U\right) V
$$

for any $V, U \in T \widetilde{M}$, where $\widetilde{\nabla}$ denotes the covariant differentiation with respect to $\widetilde{g}, \alpha^{\sharp}$ is the dual vector field of $\alpha$, the 1 form $\beta$ is defined by $\beta(X)=-\alpha(J X), \beta^{\sharp}$ is the dual vector field of $\beta$ and $T \widetilde{M}$ is the tangent bundle of $\widetilde{M}$.

An l.c.K.-manifold $\widetilde{M}(J, \widetilde{g}, \alpha)$ is called an l.c.K.-space form if it has a constant holomorphic sectional curvature. Then, [10], the Riemannian curvature tensor $\widetilde{R}$ with respect to $\widetilde{g}$ of an l.c.K.-space form with the constant holomorphic sectional curvature $c$ is given by the following formulas:

$$
\begin{gathered}
4 \widetilde{R}(X, Y, Z, W)=c\{\widetilde{g}(X, W) \widetilde{g}(Y, Z)-\widetilde{g}(X, Z) \widetilde{g}(Y, W)+ \\
+\widetilde{g}(J X, W) \widetilde{g}(J Y, Z)-\widetilde{g}(J X, Z) \widetilde{g}(J Y, W)- \\
\quad-2 \widetilde{g}(J X, Y) \widetilde{g}(J Z, W)\}+ \\
+3\{P(X, W) \widetilde{g}(Y, Z)-P(X, Z) \widetilde{g}(Y, W)+ \\
+\widetilde{g}(X, W) P(Y, Z)-\widetilde{g}(X, Z) P(Y, W)\}- \\
-\widetilde{P}(X, W) \widetilde{g}(J Y, Z)+\widetilde{P}(X, Z) \widetilde{g}(J Y, W)- \\
-\widetilde{g}(J X, W) \widetilde{P}(Y, Z)+\widetilde{g}(J X, Z) \widetilde{P}(Y, W)+ \\
+2\{\widetilde{P}(X, Y) \widetilde{g}(J Z, W)+\widetilde{g}(J X, Y) \widetilde{P}(Z, W)\}
\end{gathered}
$$

for any $X, Y, Z, W \in T \widetilde{M}$, where $P$ and $\widetilde{P}$ are respectively defined by

$$
P(X, Y)=-\left(\widetilde{\nabla}_{X} \alpha\right) Y-\alpha(X) \alpha(Y)+\frac{1}{2}\|\alpha\|^{2} \widetilde{g}(X, Y),
$$

and

$$
\widetilde{P}(X, Y)=P(J X, Y)
$$

for any $X, Y \in T \widetilde{M}$, where $\|\alpha\|$ is the length of the Lee form $\alpha$.

Let $\left(M_{1}, g_{1}\right)$ and $\left(M_{2}, g_{2}\right)$ be two Riemannian manifolds. Then we put $M=M_{1} \times M_{2}$ be the product manifold of $M_{1}$ and $M_{2}$. For a positive differentiable function $f$ on $M_{2}$, we define a Riemannian metric tensor $g$ on $M$ as

$$
g(U, V)=e^{f^{2}} g_{1}\left(\pi_{1 *} U, \pi_{1 *} V\right)+g_{2}\left(\pi_{2 *} U, \pi_{2 *} V\right)
$$

for any $U, V \in T M$, where $\pi_{1}$ (resp. $\pi_{2}$ ) denotes the projection operator of $M$ to $M_{1}\left(\operatorname{resp} . M_{2}\right)$ and $\pi_{1 *}\left(\operatorname{resp} \pi_{2 *}\right)$ is the differential of $\pi_{1}\left(\operatorname{resp} . \pi_{2}\right)$. Then the Riemannian manifold $M$ is called a warped product manifold of $M_{1}$ and $M_{2}$ with the warping function $f$ and we write it $M_{1} \otimes_{f} M_{2}$, [12]. 
Let $\nabla, \nabla_{1}$ and $\nabla_{2}$ be the covariant differentiation with respect to $g, g_{1}$ and $g_{2}$, respectively. Then, we have from (1.5)

$$
\begin{aligned}
& \nabla_{X} Y=\nabla_{1 X} Y-f^{2} e^{f^{2}} g_{1}(X, Y)\left(d_{2} \log f\right)^{*}, \\
& \nabla_{X} Z=\nabla_{Z} X=f^{2}(Z \log f) X \\
& \nabla_{Z} W=\nabla_{2 Z} W
\end{aligned}
$$

for any $X, Y \in T M_{1}$ and $Z, W \in T M_{2}$, where $d_{2} \log f$ means the differential of $\log f$ and $\left(d_{2} \log f\right)^{*}$ is the dual vector field of $d_{2} \log f$.

By virtue of (1.6), the curvature tensor $R$ with respect to $g$ is written as

$$
\begin{aligned}
R\left(X_{1}, X_{2}, X_{3}, X_{4}\right) & =e^{f^{2}}\left[R_{1}\left(X_{1}, X_{2}, X_{3}, X_{4}\right)-\right. \\
& -f^{4} e^{f^{2}}\left\|\nabla_{2} \log f\right\|^{2}\left\{g_{1}\left(X_{1}, X_{4}\right) g_{1}\left(X_{2}, X_{3}\right)-\right. \\
& \left.\left.-g_{1}\left(X_{1}, X_{3}\right) g_{1}\left(X_{2}, X_{3}\right)\right\}\right] \\
R\left(X_{1}, Z_{1}, Z_{2}, X_{2}\right) & =-f^{2} e^{f^{2}}\left\{\left(2+f^{2}\right)\left(Z_{1} \log f\right)\left(Z_{2} \log f\right)+\right. \\
& \left.+\nabla_{2 Z_{1}} \nabla_{2 Z_{2}} \log f\right\} g_{1}\left(X_{1}, X_{2}\right), \\
R\left(Z_{1}, Z_{2}, Z_{3}, Z_{4}\right) & =R_{2}\left(Z_{1}, Z_{2}, Z_{3}, Z_{4}\right), \\
\text { Other } & =0
\end{aligned}
$$

and the Ricci tensor $\rho$ with respect to $g$ is separated as

$$
\begin{aligned}
& \rho\left(X_{1}, X_{2}\right)=\rho_{1}\left(X_{1}, X_{2}\right)- \\
& \quad-f^{2} e^{f^{2}}\left\{\left(2+n_{1} f^{2}\right)\left\|\nabla_{2} \log f\right\|^{2}+\delta_{2} d_{2}\right\} g_{1}\left(X_{1}, X_{2}\right), \\
& \rho\left(X_{1}, Z_{1}\right)=0, \\
& \rho\left(Z_{1}, Z_{2}\right)=\rho_{2}\left(Z_{1}, Z_{2}\right)- \\
& \quad-n_{1} f^{2}\left\{\left(2+f^{2}\right)\left(\nabla_{2 Z_{1}} \log f\right)\left(\nabla_{2 Z_{2}} \log f\right)+\nabla_{2 Z_{1}} \nabla_{2 Z_{2}} \log f\right\},
\end{aligned}
$$

for any $X_{1}, X_{2} \in T M_{1}$ and $Z_{1}, Z_{2} \in T M_{2}$, where $R_{1}$ (resp. $R_{2}$ ) is the Riemannian curvature tensor with respect to $g_{1}$ (resp. $g_{2}$ ) and $\rho_{1}$ (resp. $\left.\rho_{2}\right)$ is the Ricci tensor with respect to $g_{1}$ (resp. $\left.g_{2}\right), d_{2}$ (resp. $\delta_{2}$ ) means the differential (resp. codifferential) with respect to $g_{2},\left\|\nabla_{2} \log f\right\|$ is the length of $\nabla_{2} \log f$ with respect to $g_{2}$ and $n_{1}=\operatorname{dim} M_{1}$.

Finally, if we respectively put $\tau, \tau_{1}$ and $\tau_{2}$ the scalar curvature with respect to $g, g_{1}$ and $g_{2}$, then from (1.8), we can easily have

$$
\tau=e^{f^{2}} \tau_{1}+\tau_{2}-\left(n_{1}-1\right) n_{1} f^{4}\left\|\nabla_{2} \log f\right\|^{2} .
$$




\section{Semi-Slant-SUbmanifolds in AN Almost Hermitian MANifold}

In general, between a Riemannian manifold $(\widetilde{M}, \widetilde{g})$ and its Riemannian submanifold $(M, g)$, we know the Gauss and Weingarten formulas

$$
\widetilde{\nabla}_{X} Y=\nabla_{X} Y+\sigma(X, Y), \quad \widetilde{\nabla}_{X} N=-A_{N} X+\nabla^{\perp}{ }_{X} N
$$

for any $X, Y \in T M$ and $N \in T^{\perp} M$, where $\nabla$ is the covariant differentiation with respect to $g, \sigma$ is the second fundamental form and $A_{N}$ is the shape operator with respect to $N$ and $\nabla^{\perp}$ is the normal connection, [6]. The second fundamental form $\sigma$ and the shape operator $A$ are related by $\widetilde{g}\left(A_{N} Y, X\right)=\widetilde{g}(\sigma(Y, X), N)$ for any $Y, X \in T M$ and $N \in T^{\perp} M$.

The Gauss equation is given by

$$
\begin{aligned}
\widetilde{R}(U, V, W, Z) & =R(U, V, W, Z)+\widetilde{g}(\sigma(U, Z), \sigma(V, W) \\
& -\widetilde{g}(\sigma(U, W), \sigma(V, Z)),
\end{aligned}
$$

for any $U, V, W, Z \in T M,[6]$.

A submanifold $M$ is said to be totally geodesic, if the second fundamental form $\sigma$ identically vanishes, [6].

We recall a warped product submanifold in a Riemannian manifold.

Let $(\widetilde{M}, \widetilde{g})$ be a Riemannian manifold. A submanifold $(M, g)$ is called a warped product submanifold of $\widetilde{M}$ if it satisfies

(i) $M$ is a product manifold of 2 submanifolds $M_{1}$ and $M_{2}$,

(ii) two submanifolds are orthogonal with respect to $\widetilde{g}$,

(iii) for certain Riemannian metric $g_{1}$ in $M_{1}, g_{2}$ in $M_{2}$ and a certain positive differentiable function $f$ in $M_{2}$, the metric tensor $g$ is defined by

$$
g(U, V)=e^{f^{2}} g_{1}\left(\pi_{1 *} U, \pi_{1 *} V\right)+g_{2}\left(\pi_{2 *} U, \pi_{2 *} V\right)
$$

for any $U, V \in T M$ is the induced metric of $\widetilde{g}$, [5].

By virtue of (1.7) and (2.3) the Riemannian curvature $\widetilde{R}$ is separated as

$$
\begin{aligned}
& \widetilde{R}\left(X_{1}, X_{2}, X_{3}, X_{4}\right)=e^{f^{2}}\left\{R_{1}\left(X_{1}, X_{2}, X_{3}, X_{4}\right)\right. \\
& \left.-f^{4} e^{f^{2}}\|\log f\|^{2} g_{1}\left(X_{1}, X_{4}\right) g_{1}\left(X_{2}, X_{3}\right)-g_{1}\left(X_{1}, X_{3}\right) g_{1}\left(X_{2}, X_{4}\right)\right\} \\
& +\widetilde{g}\left(\sigma\left(X_{1}, X_{4}\right), \sigma\left(X_{2}, X_{3}\right)\right)-\widetilde{g}\left(\sigma\left(X_{1}, X_{3}\right), \sigma\left(X_{2}, X_{4}\right)\right) \text {, } \\
& \widetilde{R}\left(X_{1}, X_{2}, X_{3}, Z_{1}\right)=\widetilde{g}\left(\sigma\left(X_{1}, Z_{1}\right), \sigma\left(X_{2}, X_{3}\right)\right)-\widetilde{g}\left(\sigma\left(X_{1}, X_{3}\right), \sigma\left(X_{2}, Z_{1}\right)\right), \\
& \widetilde{R}\left(X_{1}, X_{2}, Z_{1}, Z_{2}\right)=\widetilde{g}\left(\sigma\left(X_{1}, Z_{2}\right), \sigma\left(X_{2}, Z_{1}\right)\right)-\widetilde{g}\left(\sigma\left(X_{1}, Z_{1}\right), \sigma\left(X_{2}, Z_{2}\right)\right), \\
& \widetilde{R}\left(X_{1}, Z_{1}, Z_{2}, X_{2}\right)=-f^{2} e^{f^{2}}\left\{\left(2+f^{2}\right)\left(Z_{1} \log f\right)\left(Z_{2} \log f\right)\right. \\
& \left.+\nabla_{2 Z_{2}} \nabla_{2 Z_{1}} \log f\right\} g_{1}\left(X_{1}, X_{2}\right)+\widetilde{g}\left(\sigma\left(X_{1}, X_{2}\right), \sigma\left(Z_{1}, Z_{2}\right)\right)
\end{aligned}
$$




$$
\begin{aligned}
-\widetilde{g}\left(\sigma\left(X_{1}, Z_{2}\right), \sigma\left(Z_{1}, X_{2}\right)\right) & \\
\widetilde{R}\left(X_{1}, Z_{1}, Z_{2}, Z_{3}\right)= & \widetilde{g}\left(\sigma\left(X_{1}, Z_{3}\right), \sigma\left(Z_{1}, Z_{2}\right)\right)-\widetilde{g}\left(\sigma\left(X_{1}, Z_{2}\right), \sigma\left(Z_{1}, Z_{3}\right)\right), \\
\widetilde{R}\left(Z_{1}, Z_{2}, Z_{3}, Z_{4}\right)= & R_{2}\left(Z_{1}, Z_{2}, Z_{3}, Z_{4}\right)+ \\
& +\widetilde{g}\left(\sigma\left(Z_{1}, Z_{4}\right), \sigma\left(Z_{2}, Z_{3}\right)\right)-\widetilde{g}\left(\sigma\left(Z_{1}, Z_{3}\right), \sigma\left(Z_{2}, Z_{4}\right)\right),
\end{aligned}
$$

for any $X_{1}, X_{2}, X_{3}, X_{4} \in T M_{1}$ and $Z_{1}, Z_{2}, Z_{3}, Z_{4} \in T M_{2}$, where $R_{1}$ (resp. $R_{2}$ ) is the Riemannian curvature tensor with respect to $g_{1}$ (resp. $g_{2}$ ).

For a vector field $U \in T M$, the angle between $J U$ and $T M$ is called the Wirtingar angle of $U$.

A differentiable distribution $\mathcal{D}^{\theta}: x \rightarrow \mathcal{D}^{\theta}{ }_{x}$ on $M$ is said to be a slant one if for each $U_{x} \in \mathcal{D}^{\theta}{ }_{x}$, the Wirtingar angle of $U_{x}$ is constant $(=\theta)$ for any $x \in M$. In this case, the Wirtingar angle is said to be the slant angle. In particular, if $T M$ is slant, then the submanifold is called a slant one, [9]. A slant submanifold is holomorphic (resp. totally real) if its slant angle $\theta=0$ (resp. $\theta=\frac{\pi}{2}$ ). A slant submanifold is said to be proper if it is neither holomorphic nor totally real.

A submanifold $M$ in $\widetilde{M}$ is called a semi-slant submanifold if there exists a differentiable distribution $\mathcal{D}: x \rightarrow \mathcal{D}_{x} \subset T_{x} M$ on $M$ satisfying the following conditions:

(i) $\mathcal{D}$ is holomorphic, i.e., $J \mathcal{D}_{x}=D_{x}$ for each $x \in M$ and

(ii) the complementary orthogonal distribution $\mathcal{D}^{\theta}: x \rightarrow \mathcal{D}^{\theta}{ }_{x} \subset T_{x} M$ is slant with slant angle $\theta$, where $T_{x} M$ is the tangent vector space of $M$ at $x,[13]$.

Remark 2.1. A semi-slant submanifold is a $C R$-submanifold if the slant angle is equal to $\frac{\pi}{2},[1],[2],[3],[7],[8]$, etc.

A semi-slant submanifold $M$ is said to be proper if it is neither $C R$-, holomorphic, nor totally real.

In a submanifold $M$ of an almost Hermitian manifold $\widetilde{M}(J, \widetilde{g})$, for any $U \in T M$ and $\xi \in T^{\perp} M$, we write

$$
J U=T U+F U, \quad J \xi=t \xi+h \xi,
$$

where $T U$ (resp. $F U$ ) means the tangential (resp. normal) component of $J U$ and $t \xi$ (resp. $h \xi$ ) means the tangential (resp. normal) component of $J \xi$.

For a semi-slant submanifold $M$ of an almost Hermitian manifold $\widetilde{M}$, the tangent bundle $T M$ and the normal bundle $T^{\perp} M$ of $M$ are decomposed as

$$
T M=\mathcal{D} \oplus \mathcal{D}^{\theta}, \quad T^{\perp} M 7=F \mathcal{D}^{\theta} \oplus \nu,
$$


where $\nu$ denotes the orthogonal complementary distribution of $F \mathcal{D}^{\theta}$ in $T^{\perp} M$.

Further, in a semi-slant submanifold $M$ we write

$$
U=T_{1} U+T_{2} U
$$

for any $U \in T M$, where $T_{1} U$ (resp. $\left.T_{2} U\right)$ denotes the $\mathcal{D}\left(\right.$ resp. $\left.\mathcal{D}^{\theta}\right)$ component of $U$.

By virtue of (2.7) and (2.7), we can write

$$
J U=J T_{1} U+T T_{2} U+F T_{2} U,
$$

where $J T_{1} U \in \mathcal{D}, T T_{2} U \in \mathcal{D}^{\theta}$ and $F T_{2} U \in F \mathcal{D}^{\theta} \subset T^{\perp} M$. Thus if we put

$$
Q U=J T_{1} U+T T_{2} U
$$

for any $U \in T M$, then $Q$ is an automorphism on $T M$.

The covariant differentiation $\bar{\nabla}$ of $T_{1}, T_{2}, T, F, t$ and $h$ are defined as

$$
\begin{aligned}
\left(\bar{\nabla}_{U} T_{1}\right) V & =\nabla_{U}\left(T_{1} V\right)-T_{1} \nabla_{U} V \\
\left(\bar{\nabla}_{U} T_{2}\right) V & =\nabla_{U}\left(T_{2} V\right)-T_{2} \nabla_{U} V \\
\left(\bar{\nabla}_{U} T\right) V & =\nabla_{U}(T V)-T \nabla_{U} V \\
\left(\bar{\nabla}_{U} F\right) V & =\nabla_{U}^{\perp}(F V)-F \nabla_{U} V \\
\left(\bar{\nabla}_{U} t\right) \xi & =\nabla_{U}(t \xi)-t \nabla_{U}^{\perp} \xi \\
\left(\bar{\nabla}_{U} h\right) \xi & =\nabla_{U}^{\perp}(h \xi)-h \nabla_{U}^{\perp} \xi
\end{aligned}
$$

for any $U, V \in T M$ and $\xi \in T^{\perp} M$.

Moreover, if we define the covariant differentiation $\bar{\nabla}$ of $Q$

$$
\left(\bar{\nabla}_{U} Q\right) V=\nabla_{U}(Q V)-Q \nabla_{U} V
$$

for any $U, V \in T M$, then using (2.10), we have

$$
\begin{aligned}
\left(\bar{\nabla}_{U} Q\right) V & =\left(\widetilde{\nabla}_{U} J\right) T_{1} V+J\left(\bar{\nabla}_{U} T_{1}\right) V+\left(\bar{\nabla}_{U} T\right)\left(T_{2} V\right) \\
& +T\left(\bar{\nabla}_{U} T_{2}\right) V+J \sigma\left(U, T_{1} V\right)-\sigma\left(U, J T_{1} V\right)
\end{aligned}
$$

for any $U, V \in T M$. In particular, for any $X, Y \in \mathcal{D}$, the equation (2.12) is written as

$$
\left(\bar{\nabla}_{X} Q\right) Y=\left(\widetilde{\nabla}_{X} J\right) Y+F T_{2} \nabla_{X} Y+t \sigma(X, Y)+h \sigma(X, Y)-\sigma(X, T Y) .
$$

Now, for $U, V \in T M$, we write

$$
\left(\widetilde{\nabla}_{U} J\right) V=\mathcal{P}_{U} V+\mathcal{Q}_{U} V
$$

where $\mathcal{P}_{U} V$ (resp. $\mathcal{Q}_{U} V$ ) denotes the tangential (resp. normal) part of $\left(\widetilde{\nabla}_{U} J\right) V$. 


\section{SEMI-SLANT SUBMANIFOLDS IN AN L.C.K.-MANIFOLD}

Let $M$ be a semi-slant submanifold of an l.c.K.-manifold $\widetilde{M}(J, \widetilde{g}, \alpha)$. Then we have from (1.1) and (2.14)

$$
\begin{aligned}
\mathcal{P}_{U} V & =-\widetilde{g}\left(\alpha_{1}^{\sharp}, V\right) T U+\widetilde{g}(U, V)\left(T \alpha_{1}^{\sharp}+t \alpha_{2}^{\sharp}\right)+\widetilde{g}(T U, V) \alpha_{1}^{\sharp} \\
& -\widetilde{g}\left(T \alpha_{1}^{\sharp}+t \alpha_{2}^{\sharp}, V\right) U, \\
\mathcal{Q}_{U} V & =-\widetilde{g}\left(\alpha_{1}^{\sharp}, V\right) F U+\widetilde{g}(U, V)\left(F \alpha_{1}^{\sharp}+h \alpha_{2}^{\sharp}\right)+\widetilde{g}(T U, V) \alpha_{2}^{\sharp},
\end{aligned}
$$

where $\alpha_{1}^{\sharp}$ (resp. $\alpha_{2}^{\sharp}$ ) means the tangential (resp. normal) component of $\alpha^{\sharp}$.

In a semi-slant submanifold in an l.c.K.-manifold, we have from $(3.1)_{2}$

$$
\mathcal{Q}_{X} Y-\mathcal{Q}_{Y} X=2 \widetilde{g}(T X, Y) \alpha_{2}^{\sharp}
$$

for any $X, Y \in \mathcal{D}$.

Using theorems of V. A. Khan and M. A. Khan on integrability of the distributions $\mathcal{D}$ and $\mathcal{D}^{\theta}$ of a semi-slant submanifold in an almost Hermitian manifold, in [4], we proved

Proposition 3.1. (I) The holomorphic distribution $\mathcal{D}$ of a semi-slant submanifold $M$ in an l.c.K.-manifold $\widetilde{M}(J, \widetilde{g}, \alpha)$ is integrable if and only if

$$
\sigma(X, T Y)-\sigma(Y, T X)=\mathcal{Q}_{X} Y-\mathcal{Q}_{Y} X=2 \widetilde{g}(T X, Y) \alpha_{2}^{\sharp}
$$

for any $X, Y \in \mathcal{D}$.

(II) The slant distribution $\mathcal{D}^{\theta}$ of a semi-slant submanifold $M$ in an locally conformal Kaehler manifold $\widetilde{M}(J, \widetilde{g}, \alpha)$ is integrable if and only if

$$
\begin{aligned}
& T_{1}\left(\nabla_{Z} T W-\nabla_{W} T Z+A_{F Z} W-A_{F W} Z+\right. \\
& \left.\quad+\widetilde{g}\left(\alpha_{1}^{\sharp}, W\right) T Z-\widetilde{g}\left(\alpha_{1}^{\sharp}, Z\right) T W+2 \widetilde{g}(T W, Z) \alpha_{1}^{\sharp}\right)=0
\end{aligned}
$$

or equivalently

$$
\begin{aligned}
T_{1}\left\{\left(\bar{\nabla}_{Z} T\right) W-\left(\bar{\nabla}_{W} T\right) Z+T[Z, W]+A_{F Z} W-A_{F W} Z+\right. \\
\left.+\widetilde{g}\left(\alpha_{1}^{\sharp}, W\right) T Z-\widetilde{g}\left(\alpha_{1}^{\sharp}, Z\right) T W+2 \widetilde{g}(T W, Z) \alpha_{1}^{\sharp}\right\}=0
\end{aligned}
$$

for any $Z, W \in \mathcal{D}^{\theta}$.

4. WARPED PRODUCT SEMI-SLANT SUBMANIFOLDS IN L.C.K.-MANIFOLDS

Let $\mathcal{D}$ and $\mathcal{D}^{\theta}$ be two integrable distributions on a semi-slant submanifold $M$ of an l.c.K.-manifold $\widetilde{M}(J, \widetilde{g}, \alpha)$. Then (3.3) and (3.4) hold true. Let also $M_{\mathcal{D}}$ (resp. $M_{\mathcal{D}^{\theta}}$ ) be the maximal integral submanifold of $\mathcal{D}\left(\right.$ resp. $\left.\mathcal{D}^{\theta}\right)$. Then $M$ is a product manifold of $M_{\mathcal{D}}$ and $M_{\mathcal{D}^{\theta}}$, that is,

$$
M=M_{\mathcal{D}} \otimes M_{\mathcal{D}^{\theta}} .
$$


We call the submanifold $M_{\mathcal{D}}$ (resp. $M_{\mathcal{D}^{\theta}}$ ) the holomorphic (resp. slant) component of $M$.

We define the following two type warped product submanifolds

$$
M_{1}:=M_{\mathcal{D}} \otimes_{f_{1}} M_{\mathcal{D}^{\theta}}
$$

for a certain differentiable function $f_{1}$ on $M_{\mathcal{D}^{\theta}}$ and

$$
M_{2}:=M_{\mathcal{D}^{\theta}} \otimes_{f_{2}} M_{\mathcal{D}}
$$

for a certain differentiable function $f_{2}$ on $M_{\mathcal{D}}$. We say that $M_{1}$ (resp. $M_{2}$ ) the first (resp. second) type warped product semi-slant submanifold of an l.c.K.-manifold.

In this paper, we mainly consider the first type warped product semislant submanifold.

Let $M$ be the first type warped product semi-slant submanifold in an l.c.K.-manifold $\widetilde{M}$. Then the induced metric tensor $g$ on $M$ from $\widetilde{M}$ is given by

$$
g(U, V)=e^{f_{1}^{2}} g_{\mathcal{D}}\left(\pi_{\mathcal{D}} * U, \pi_{\mathcal{D}} * V\right)+g_{\mathcal{D}^{\theta}}\left(\pi_{\mathcal{D}^{\theta}} * U, \pi_{\mathcal{D}^{\theta}} * V\right)
$$

for any $U, V \in T M$, where $g_{\mathcal{D}}$ (resp. $g_{\mathcal{D}^{\theta}}$ ) denotes the Riemannian metric on $M_{\mathcal{D}}$ (resp. $\left.M_{\mathcal{D}^{\theta}}\right), \pi_{\mathcal{D}}$ (resp. $\pi_{\mathcal{D}^{\theta}}$ ) is the projection operator of $M$ to $M_{\mathcal{D}}$ (resp. $\quad M_{\mathcal{D}^{\theta}}$ ) and $f_{1}$ is a certain positive differentiable function on $M_{\mathcal{D}^{\theta}}$. Now, we denote by $\widetilde{\nabla}, \nabla, \nabla^{\mathcal{D}}$ and $\nabla^{\mathcal{D}^{\theta}}$ the covariant differentiations with respect to $\widetilde{g}, g, g_{\mathcal{D}}$ and $g_{\mathcal{D}^{\theta}}$, respectively. Since we have from (1.6)

$$
\begin{aligned}
\nabla_{X} Y & =\nabla^{\mathcal{D}}{ }_{X} Y-f_{1}^{2} e^{f_{1}^{2}}\left(d_{1} \log f_{1}\right)^{*} g_{\mathcal{D}}(X, Y), \\
\nabla_{X} Z & =\nabla_{Z} X=f_{1}^{2}\left(Z \log f_{1}\right) X, \\
\nabla_{Z} W & =\nabla^{\mathcal{D}^{\theta}}{ }_{Z} W
\end{aligned}
$$

for any $X, Y \in \mathcal{D}$ and $Z, W \in \mathcal{D}^{\theta}$, where we put $\left(d_{1} \log f_{1}\right)$ is the differential of $\log f_{1}$ with respect to $g_{\mathcal{D}^{\theta}}$.

Using Gauss formula and the above equation, we obtain

$$
\begin{aligned}
\widetilde{\nabla}_{X} Y & =\nabla^{\mathcal{D}}{ }_{Y} X-f_{1}^{2} e^{f_{1}^{2}}\left(d_{1} \log f_{1}\right)^{*} g_{\mathcal{D}}(X, Y)+\sigma(X, Y), \\
\widetilde{\nabla}_{X} Z & =\widetilde{\nabla}_{Z} X=f_{1}^{2}\left(Z \log f_{1}\right) X+\sigma(X, Z), \\
\widetilde{\nabla}_{Z} W & =\nabla^{\mathcal{D}^{\theta}}{ }_{Z} W+\sigma(Z, W)
\end{aligned}
$$

for any $X, Y \in \mathcal{D}$ and $Z, W \in \mathcal{D}^{\theta}$.

Due to (4.6) between the Riemannian curvature tensors

- $R\left(U_{1}, U_{2}, U_{3}, U_{4}\right)$ with respect to $g$,

- $R^{\mathcal{D}}\left(X_{1}, X_{2}, X_{3}, X_{4}\right)$ with respect to $g_{\mathcal{D}}$, and

- $R^{\mathcal{D}^{\theta}}\left(Z_{1}, Z_{2}, Z_{3}, Z_{4}\right)$ with respect to $g_{\mathcal{D}^{\theta}}$, 
we know the following relations:

$$
\begin{aligned}
& R\left(X_{1}, X_{2}, X_{3}, X_{4}\right)=e^{f_{1}^{2}}\left[R^{\mathcal{D}}\left(X_{1}, X_{2}, X_{3}, X_{4}\right)\right. \\
& -f_{1}^{4} e^{f_{1}^{2}}\left\|\nabla^{\mathcal{D}^{\theta}} \log f_{1}\right\|^{2}\left\{g_{\mathcal{D}}\left(X_{1}, X_{4}\right) g_{\mathcal{D}}\left(X_{2}, X_{3}\right)\right. \\
& \left.\left.-g_{\mathcal{D}}\left(X_{1}, X_{3}\right) g_{\mathcal{D}}\left(X_{2}, X_{4}\right)\right\}\right] \text {, } \\
& R\left(X_{1}, Z_{1}, Z_{2}, X_{2}\right)=-f_{1}^{2} e^{f_{1}^{2}}\left\{\left(2+f_{1}^{2}\right)\left(Z_{1} \log f_{1}\right)\left(Z_{2} \log f_{1}\right)\right. \\
& \left.+\nabla^{\mathcal{D}^{\theta}}{ }_{Z_{1}} \nabla^{\mathcal{D}^{\theta}} Z_{2} \log f_{1}\right\} g_{\mathcal{D}}\left(X_{1}, X_{2}\right), \\
& R\left(Z_{1}, Z_{2}, Z_{3}, Z_{4}\right)=R^{\mathcal{D}^{\theta}}\left(Z_{1}, Z_{2}, Z_{3}, Z_{4}\right), \\
& \text { Others }=0 \text {, }
\end{aligned}
$$

for any $X_{1}, X_{2}, X_{3}, X_{4} \in \mathcal{D}$ and $Z_{1}, Z_{2}, Z_{3}, Z_{4} \in \mathcal{D}^{\theta}$.

By virtue of the above equation and the Gauss equation, we have the following

$$
\begin{aligned}
& \widetilde{R}\left(X_{1}, X_{2}, X_{3}, X_{4}\right)=e^{f_{1}^{2}}\left[R^{\mathcal{D}}\left(X_{1}, X_{2}, X_{3}, X_{4}\right)\right. \\
& -f_{1}^{4} e^{f_{1}^{2}}\left\|\nabla^{\mathcal{D}^{\theta}} \log f_{1}\right\|^{2}\left\{g_{\mathcal{D}}\left(X_{1}, X_{4}\right) g_{\mathcal{D}}\left(X_{2}, X_{3}\right)\right. \\
& \left.\left.-g_{\mathcal{D}}\left(X_{1}, X_{3}\right) g_{\mathcal{D}}\left(X_{2}, X_{4}\right)\right\}\right]+\widetilde{g}\left(\sigma\left(X_{1}, X_{4}\right), \sigma\left(X_{2}, X_{3}\right)\right) \\
& -\widetilde{g}\left(\sigma\left(X_{1}, X_{3}\right), \sigma\left(X_{2}, X_{4}\right)\right) \text {, } \\
& \widetilde{R}\left(X_{1}, X_{2}, X_{3}, Z_{1}\right)=\widetilde{g}\left(\sigma\left(X_{1}, Z_{1}\right), \sigma\left(X_{2}, X_{3}\right)\right)- \\
& -\widetilde{g}\left(\sigma\left(X_{1}, X_{3}\right), \sigma\left(X_{2}, Z_{1}\right)\right), \\
& \widetilde{R}\left(X_{1}, X_{2}, Z_{1}, Z_{2}\right)=\widetilde{g}\left(\sigma\left(X_{1}, Z_{2}\right), \sigma\left(X_{2}, Z_{1}\right)\right)- \\
& -\widetilde{g}\left(\sigma\left(X_{1}, Z_{1}\right), \sigma\left(X_{2}, Z_{2}\right)\right), \\
& \widetilde{R}\left(X_{1}, Z_{1}, Z_{2}, Z_{3}\right)=\widetilde{g}\left(\sigma\left(X_{1}, Z_{3}\right), \sigma\left(Z_{1}, Z_{2}\right)\right)- \\
& -\widetilde{g}\left(\sigma\left(X_{1}, Z_{2}\right), \sigma\left(Z_{1}, Z_{3}\right)\right), \\
& \widetilde{R}\left(X_{1}, Z_{1}, Z_{2}, X_{2}\right)=-f_{1}^{2} e^{f_{1}^{2}}\left\{\left(2+f_{1}^{2}\right)\left(Z_{1} \log f_{1}\right)\left(Z_{2} \log f_{1}\right)\right. \\
& \begin{aligned}
&\left.+\nabla^{\mathcal{D}^{\theta}}{ }_{Z_{1}} \nabla^{\mathcal{D}^{\theta}}{ }_{2} \log f_{1}\right\} g_{\mathcal{D}}\left(X_{1}, X_{2}\right) \\
&+\widetilde{g}\left(\sigma\left(X_{1}, X_{2}\right), \sigma\left(Z_{1}, Z_{2}\right)\right)-\widetilde{g}\left(\sigma\left(X_{1}, Z_{2}\right), \sigma\left(Z_{1}, X_{2}\right)\right), \\
& \widetilde{R}\left(Z_{1}, Z_{2}, Z_{3}, Z_{4}\right)=R^{\mathcal{D}^{\theta}}\left(Z_{1}, Z_{2}, Z_{3}, Z_{4}\right)+\widetilde{g}\left(\sigma\left(Z_{1}, Z_{4}\right), \sigma\left(Z_{2}, Z_{3}\right)\right)
\end{aligned} \\
& \begin{aligned}
&\left.+\nabla^{\mathcal{D}^{\theta}}{ }_{Z_{1}} \nabla^{\mathcal{D}^{\theta}}{ }_{2} \log f_{1}\right\} g_{\mathcal{D}}\left(X_{1}, X_{2}\right) \\
&+\widetilde{g}\left(\sigma\left(X_{1}, X_{2}\right), \sigma\left(Z_{1}, Z_{2}\right)\right)-\widetilde{g}\left(\sigma\left(X_{1}, Z_{2}\right), \sigma\left(Z_{1}, X_{2}\right)\right), \\
& \widetilde{R}\left(Z_{1}, Z_{2}, Z_{3}, Z_{4}\right)=R^{\mathcal{D}^{\theta}}\left(Z_{1}, Z_{2}, Z_{3}, Z_{4}\right)+\widetilde{g}\left(\sigma\left(Z_{1}, Z_{4}\right), \sigma\left(Z_{2}, Z_{3}\right)\right)
\end{aligned} \\
& \begin{aligned}
&\left.+\nabla^{\mathcal{D}^{\theta}}{ }_{Z} \nabla^{\mathcal{D}^{\theta}}{ }_{2} \log f_{1}\right\} g_{\mathcal{D}}\left(X_{1}, X_{2}\right) \\
&+\widetilde{g}\left(\sigma\left(X_{1}, X_{2}\right), \sigma\left(Z_{1}, Z_{2}\right)\right)-\widetilde{g}\left(\sigma\left(X_{1}, Z_{2}\right), \sigma\left(Z_{1}, X_{2}\right)\right), \\
& \widetilde{R}\left(Z_{1}, Z_{2}, Z_{3}, Z_{4}\right)=R^{\mathcal{D}^{\theta}}\left(Z_{1}, Z_{2}, Z_{3}, Z_{4}\right)+\widetilde{g}\left(\sigma\left(Z_{1}, Z_{4}\right), \sigma\left(Z_{2}, Z_{3}\right)\right)
\end{aligned} \\
& -\widetilde{g}\left(\sigma\left(Z_{1}, Z_{3}\right), \sigma\left(Z_{2}, Z_{4}\right)\right),
\end{aligned}
$$

for any $X_{1}, X_{2}, X_{3}, X_{4} \in \mathcal{D}$ and $Z_{1}, Z_{2}, Z_{3}, Z_{4} \in \mathcal{D}^{\theta}$. 
Next, we assume that our ambient manifold is an l.c.K.-space form. Then the curvature tensor $\widetilde{R}$ satisfies (1.2). Using this, we can separate the curvature tensor $\widetilde{R}$ as

$$
\begin{aligned}
& 4 \widetilde{R}\left(X_{1}, X_{2}, X_{3}, X_{4}\right)=c\left\{\widetilde{g}\left(X_{1}, X_{4}\right) \widetilde{g}\left(X_{2}, X_{3}\right)-\widetilde{g}\left(X_{1}, X_{3}\right) \widetilde{g}\left(X_{2}, X_{4}\right)+\right. \\
& +\widetilde{g}\left(T X_{1}, X_{4}\right) \widetilde{g}\left(T X_{2}, X_{3}\right)-\widetilde{g}\left(T X_{1}, X_{3}\right) \widetilde{g}\left(T X_{2}, X_{4}\right)- \\
& \left.-2 \widetilde{g}\left(T X_{1}, X_{2}\right) \widetilde{g}\left(T X_{3}, X_{4}\right)\right\}+ \\
& +3\left\{P\left(X_{1}, X_{4}\right) \widetilde{g}\left(X_{2}, X_{3}\right)-P\left(X_{1}, X_{3}\right) \widetilde{g}\left(X_{2}, X_{4}\right)+\right. \\
& \left.+P\left(X_{2}, X_{3}\right) \widetilde{g}\left(X_{1}, X_{4}\right)-P\left(X_{2}, X_{4}\right) \widetilde{g}\left(X_{1}, X\right)\right\}- \\
& -\widetilde{P}\left(X_{1}, X_{4}\right) \widetilde{g}\left(T X_{2}, X_{3}\right)+\widetilde{P}\left(X_{1}, X_{3}\right) \widetilde{g}\left(T X_{2}, X_{4}\right)- \\
& \text { - } \widetilde{P}\left(X_{2}, X_{3}\right) \widetilde{g}\left(T X_{1}, X_{4}\right)+\widetilde{P}\left(X_{2}, X_{4}\right) \widetilde{g}\left(T X_{1}, X_{3}\right)+ \\
& +2\left\{\widetilde{P}\left(X_{1}, X_{2}\right) \widetilde{g}\left(T X_{3}, X_{4}\right)+\widetilde{P}\left(X_{3}, X_{4}\right) \widetilde{g}\left(T X_{1}, X_{2}\right)\right\}, \\
& 4 \widetilde{R}\left(X_{1}, X_{2}, X_{3}, Z_{1}\right)=3\left\{P\left(X_{1}, Z_{1}\right) \widetilde{g}\left(X_{2}, X_{3}\right)-P\left(X_{2}, Z_{1}\right) \widetilde{g}\left(X_{1}, X_{3}\right)\right\} \\
& \text { - } \widetilde{P}\left(X_{1}, Z_{1}\right) \widetilde{g}\left(T X_{2}, X_{3}\right)+\widetilde{P}\left(X_{2}, Z_{1}\right) \widetilde{g}\left(T X_{1}, X_{3}\right) \\
& +2 \widetilde{P}\left(X_{3}, Z_{1}\right) \widetilde{g}\left(T X_{1}, X_{2}\right), \\
& 2 \widetilde{R}\left(X_{1}, X_{2}, Z_{1}, Z_{2}\right)=-c \widetilde{g}\left(T X_{1}, X_{2}\right) \widetilde{g}\left(T Z_{1}, Z_{2}\right) \\
& +\widetilde{P}\left(X_{1}, X_{2}\right) \widetilde{g}\left(T Z_{1}, Z_{2}\right)+\widetilde{P}\left(Z_{1}, Z_{2}\right) \widetilde{g}\left(T X_{1}, X_{2}\right), \\
& 4 \widetilde{R}\left(X_{1}, Z_{1}, Z_{2}, X_{2}\right)=c\left\{\widetilde{g}\left(X_{1}, X_{2}\right) \widetilde{g}\left(Z_{1}, Z_{2}\right)+\widetilde{g}\left(T X_{1}, X_{2}\right) \widetilde{g}\left(T Z_{1}, Z_{2}\right)\right\} \\
& +3\left\{P\left(X_{1}, X_{2}\right) \widetilde{g}\left(Z_{1}, Z_{2}\right)+P\left(Z_{1}, Z_{2}\right) \widetilde{g}\left(X_{1}, X_{2}\right)\right\} \\
& \text { - } \widetilde{P}\left(X_{1}, X_{2}\right) \widetilde{g}\left(T Z_{1}, Z_{2}\right)-\widetilde{P}\left(Z_{1}, Z_{2}\right) \widetilde{g}\left(T X_{1}, X_{2}\right) \text {, } \\
& 4 \widetilde{R}\left(X_{1}, Z_{1}, Z_{2}, Z_{3}\right)=3\left\{P\left(X_{1}, Z_{3}\right) \widetilde{g}\left(Z_{1}, Z_{2}\right)-P\left(X_{1}, Z_{2}\right) \widetilde{g}\left(Z_{1}, Z_{3}\right)\right\} \\
& \text { - } \widetilde{P}\left(X_{1}, Z_{3}\right) \widetilde{g}\left(T Z_{1}, Z_{2}\right)+\widetilde{P}\left(X_{1}, Z_{2}\right) \widetilde{g}\left(T Z_{1}, Z_{3}\right) \\
& +2 \widetilde{P}\left(X_{1}, Z_{1}\right) \widetilde{g}\left(T Z_{2}, Z_{3}\right), \\
& +\widetilde{g}\left(T Z_{1}, Z_{4}\right) \widetilde{g}\left(T Z_{2}, Z_{3}\right)-\widetilde{g}\left(T Z_{1}, Z_{3}\right) \widetilde{g}\left(T Z_{2}, Z_{4}\right)- \\
& \left.-2 \widetilde{g}\left(T Z_{1}, Z_{2}\right) \widetilde{g}\left(T Z_{3}, X_{4}\right)\right\}+ \\
& +3\left\{P\left(Z_{1}, Z_{4}\right) \widetilde{g}\left(Z_{2}, Z_{3}\right)-P\left(Z_{1}, Z_{3}\right) \widetilde{g}\left(Z_{2}, Z_{4}\right)-\right. \\
& \left.+P\left(Z_{2}, Z_{3}\right) \widetilde{g}\left(Z_{1}, Z_{4}\right)\right\}- \\
& \text { - } \widetilde{P}\left(Z_{1}, Z_{4}\right) \widetilde{g}\left(T Z_{2}, Z_{3}\right)+\widetilde{P}\left(Z_{1}, Z_{3}\right) \widetilde{g}\left(T Z_{2}, Z_{4}\right)-
\end{aligned}
$$




$$
\begin{aligned}
& -\widetilde{P}\left(Z_{2}, Z_{3}\right) \widetilde{g}\left(T Z_{1}, Z_{4}\right)+\widetilde{P}\left(Z_{2}, Z_{4}\right) \widetilde{g}\left(T Z_{1}, Z_{3}\right)+ \\
& +2\left\{\widetilde{P}\left(Z_{1}, Z_{2}\right) \widetilde{g}\left(T Z_{3}, Z_{4}\right)+\widetilde{P}\left(Z_{3}, Z_{4}\right) \widetilde{g}\left(T Z_{1}, Z_{2}\right)\right\}
\end{aligned}
$$

for any $X_{1}, X_{2}, X_{3}, X_{4} \in \mathcal{D}$ and $Z_{1}, Z_{2}, Z_{3}, Z_{4} \in \mathcal{D}^{\theta}$.

Thus we have from (4.8) and (4.9) that

$$
\begin{aligned}
& 4\left\{\widetilde{g}\left(\sigma\left(X_{1}, X_{4}\right), \sigma\left(X_{2}, X_{3}\right)\right)-\widetilde{g}\left(\sigma\left(X_{1}, X_{3}\right), \sigma\left(X_{2}, X_{4}\right)\right)\right\}= \\
& -4 e^{f_{1}^{2}} R^{\mathcal{D}}\left(X_{1}, X_{2}, X_{3}, X_{4}\right) \\
& +\left\{c+4 f_{1}^{2}\left\|\nabla^{\mathcal{D}^{\theta}} \log f_{1}\right\|^{2}\right\}\left\{\widetilde{g}\left(X_{1}, X_{4}\right) \widetilde{g}\left(X_{2}, X_{3}\right)\right. \\
& \left.-\widetilde{g}\left(X_{1}, X_{3}\right) \widetilde{g}\left(X_{2}, X_{4}\right)\right\}+c\left\{\widetilde{g}\left(T X_{1}, X_{4}\right) \widetilde{g}\left(T X_{2}, X_{3}\right)\right. \\
& \left.-\widetilde{g}\left(T X_{1}, X_{3}\right) \widetilde{g}\left(T X_{2}, X_{4}\right)-2 \widetilde{g}\left(T X_{1}, X_{2}\right) \widetilde{g}\left(T X_{3}, X_{4}\right)\right\} \\
& +3\left\{P\left(X_{1}, X_{4}\right) \widetilde{g}\left(X_{2}, X_{3}\right)-P\left(X_{1}, X_{3}\right) \widetilde{g}\left(X_{2}, X_{4}\right)\right. \\
& \left.+P\left(X_{2}, X_{3}\right) \widetilde{g}\left(X_{1}, X_{4}\right)-P\left(X_{2}, X_{4}\right) \widetilde{g}\left(X_{1}, X_{3}\right)\right\} \\
& \text { - } \widetilde{P}\left(X_{1}, X_{4}\right) \widetilde{g}\left(T X_{2}, X_{3}\right)+\widetilde{P}\left(X_{1}, X_{3}\right) \widetilde{g}\left(T X_{2}, X_{4}\right) \\
& \text { - } \widetilde{P}\left(X_{2}, X_{3}\right) \widetilde{g}\left(T X_{1}, X_{4}\right)+\widetilde{P}\left(X_{2}, X_{4}\right) \widetilde{g}\left(T X_{1}, X_{3}\right) \\
& +2\left\{\widetilde{P}\left(X_{1}, X_{2}\right) \widetilde{g}\left(T X_{3}, X_{4}\right)+\widetilde{P}\left(X_{3}, X_{4}\right) \widetilde{g}\left(T X_{1}, X_{2}\right)\right\}, \\
& 4\left\{\widetilde{g}\left(\sigma\left(X_{1}, Z_{1}\right), \sigma\left(X_{2}, X_{3}\right)\right)-\widetilde{g}\left(\sigma\left(X_{1}, X_{3}\right), \sigma\left(X_{2}, Z_{1}\right)\right)\right\}= \\
& 3\left\{P\left(X_{1}, Z_{1}\right) \widetilde{g}\left(X_{2}, X_{3}\right)-P\left(X_{2}, Z_{1}\right) \widetilde{g}\left(X_{1}, X_{3}\right)\right\} \\
& \text { - } \widetilde{P}\left(X_{1}, Z_{1}\right) \widetilde{g}\left(T X_{2}, X_{3}\right)+\widetilde{P}\left(X_{2}, Z_{1}\right) \widetilde{g}\left(T X_{1}, X_{3}\right) \\
& +2 \widetilde{P}\left(X_{3}, Z_{1}\right) \widetilde{g}\left(T X_{1}, X_{2}\right), \\
& 2\left\{\widetilde{g}\left(\sigma\left(X_{1}, Z_{2}\right), \sigma\left(X_{2}, Z_{1}\right)\right)-\widetilde{g}\left(\sigma\left(X_{1}, Z_{1}\right), \sigma\left(X_{2}, Z_{2}\right)\right)\right\}= \\
& -c \widetilde{g}\left(T X_{1}, X_{2}\right) \widetilde{g}\left(T Z_{1}, Z_{2}\right)+\widetilde{P}\left(X_{1}, X_{2}\right) \widetilde{g}\left(T Z_{1}, Z_{2}\right) \\
& +\widetilde{P}\left(Z_{1}, Z_{2}\right) \widetilde{g}\left(T X_{1}, X_{2}\right), \\
& 4\left\{\widetilde{g}\left(\sigma\left(X_{1}, X_{2}\right), \sigma\left(Z_{1}, Z_{2}\right)\right)-\widetilde{g}\left(\sigma\left(X_{1}, Z_{2}\right), \sigma\left(Z_{1}, X_{2}\right)\right)\right\}= \\
& 4 f_{1}^{2} e^{f_{1}^{2}}\left\{\left(2+f_{1}^{2}\right)\left(Z_{1} \log f_{1}\right)\left(Z_{2} \log f_{1}\right)\right. \\
& \left.+\nabla^{\mathcal{D}^{\theta}} Z_{1} \nabla^{\mathcal{D}^{\theta}} Z_{2} \log f_{1}\right\} g_{\mathcal{D}}\left(X_{1}, X_{2}\right) \\
& +c\left\{\widetilde{g}\left(X_{1}, X_{2}\right) \widetilde{g}\left(Z_{1}, Z_{2}\right)+\widetilde{g}\left(T X_{1}, X_{2}\right) \widetilde{g}\left(T Z_{1}, Z_{2}\right)\right\} \\
& +3\left\{P\left(X_{1}, X_{2}\right) \widetilde{g}\left(Z_{1}, Z_{2}\right)+P\left(Z_{1}, Z_{2}\right) \widetilde{g}\left(X_{1}, X_{2}\right)\right\} \\
& \text { - } \widetilde{P}\left(X_{1}, X_{2}\right) \widetilde{g}\left(T Z_{1}, Z_{2}\right)-\widetilde{P}\left(Z_{1}, Z_{2}\right) \widetilde{g}\left(T X_{1}, X_{2}\right), \\
& 4\left\{\widetilde{g}\left(\sigma\left(X_{1}, Z_{3}\right), \sigma\left(Z_{1}, Z_{2}\right)\right)-\widetilde{g}\left(\sigma\left(X_{1}, Z_{2}\right), \sigma\left(Z_{1}, Z_{3}\right)\right)\right\}= \\
& 3\left\{P\left(X_{1}, Z_{3}\right) \widetilde{g}\left(Z_{1}, Z_{2}\right)-P\left(X_{1}, Z_{2}\right) \widetilde{g}\left(Z_{1}, Z_{3}\right)\right\}
\end{aligned}
$$


Warped product semi-slant submanifolds

$$
\begin{aligned}
&-\widetilde{P}\left(X_{1}, Z_{3}\right) \widetilde{g}\left(T Z_{1}, Z_{2}\right)+\widetilde{P}\left(X_{1}, Z_{2}\right) \widetilde{g}\left(T Z_{1}, Z_{3}\right) \\
&+2 \widetilde{P}\left(X_{1}, Z_{1}\right) \widetilde{g}\left(T Z_{2}, Z_{3}\right), \\
& 4\left\{\widetilde{g}\left(\sigma\left(Z_{1}, Z_{4}\right), \sigma\left(Z_{2}, Z_{3}\right)\right)-\widetilde{g}\left(\sigma\left(Z_{1}, Z_{3}\right), \sigma\left(Z_{2}, Z_{4}\right)\right)\right\}= \\
& \quad-4 R^{\mathcal{D}^{\theta}}\left(Z_{1}, Z_{2}, Z_{3}, Z_{4}\right) \\
& \quad+c\left\{\widetilde{g}\left(Z_{1}, Z_{4}\right) \widetilde{g}\left(Z_{2}, Z_{3}\right)-\widetilde{g}\left(Z_{1}, Z_{3}\right) \widetilde{g}\left(Z_{2}, Z_{4}\right)\right. \\
& \quad+\widetilde{g}\left(T Z_{1}, Z_{4}\right) \widetilde{g}\left(T Z_{2}, Z_{3}\right)-\widetilde{g}\left(T Z_{1}, Z_{3}\right) \widetilde{g}\left(T Z_{2}, Z_{4}\right) \\
&\left.\quad-2 \widetilde{g}\left(T Z_{1}, Z_{2}\right) \widetilde{g}\left(T Z_{3}, X_{4}\right)\right\}+3\left\{P\left(Z_{1}, Z_{4}\right) \widetilde{g}\left(Z_{2}, Z_{3}\right)\right. \\
& \quad-P\left(Z_{1}, Z_{3}\right) \widetilde{g}\left(Z_{2}, Z_{4}\right)+P\left(Z_{2}, Z_{3}\right) \widetilde{g}\left(Z_{1}, Z_{4}\right) \\
& \\
&+\left(Z_{2}, Z_{4}\right) \widetilde{g}\left(Z_{1}, Z_{3}\right\}-\widetilde{P}\left(Z_{1}, Z_{4}\right) \widetilde{g}\left(T Z_{2}, Z_{3}\right) \\
&+\widetilde{P}\left(Z_{1}, Z_{3}\right) \widetilde{g}\left(T Z_{2}, Z_{4}\right)-\widetilde{P}\left(Z_{2}, Z_{3}\right) \widetilde{g}\left(T Z_{1}, Z_{4}\right) \\
&+\widetilde{P}\left(Z_{2}, Z_{4}\right) \widetilde{g}\left(T Z_{1}, Z_{3}\right)+2\left\{\widetilde{P}\left(Z_{1}, Z_{2}\right) \widetilde{g}\left(T Z_{3}, Z_{4}\right)\right. \\
&\left.+\widetilde{P}\left(Z_{3}, Z_{4}\right) \widetilde{g}\left(T Z_{1}, Z_{2}\right)\right\},
\end{aligned}
$$

for any $X_{1}, X_{2}, X_{3}, X_{4} \in \mathcal{D}$ and $Z_{1}, Z_{2}, Z_{3}, Z_{4} \in \mathcal{D}^{\theta}$. Let

$$
\begin{aligned}
& e_{1}, \ldots, e_{p}, e_{1}{ }^{*}, \ldots, e_{p}{ }^{*}, \\
& e_{2 p+1}, \ldots e_{2 p+q}, e_{2 p+1}{ }^{*}, \ldots, e_{2 p+q}{ }^{*}, \\
& e_{n+q+1}, \ldots, e_{n+q+s}, e_{n+q+1}{ }^{*}, \ldots, e_{n+q+s}{ }^{*}
\end{aligned}
$$

be a generalized adapted local frame of $\widetilde{M}$, [4].

Using this frame, the Gauss equation (4.10) is written as

$$
\begin{aligned}
& 4\left\{\widetilde{g}\left(\sigma_{k h}, \sigma_{j i}\right)-\widetilde{g}\left(\sigma_{k i}, \sigma_{j h}\right)\right\}= \\
& -4 e^{f_{1}^{2}} R^{\mathcal{D}}{ }_{k j i h}+f_{1}^{2}\left\|\nabla^{\mathcal{D}^{\theta}} \log f_{1}\right\|^{2}\left(\delta_{k h} \delta_{j i}-\delta_{k i} \delta_{j h}\right) \\
& -3\left(P_{k h} \delta_{j i}-P_{k i} \delta_{j h}+P_{j i} \delta_{k h}-P_{j h} \delta_{k i}\right) \\
& +P\left(J e_{k}, e_{h}\right) \widetilde{g}\left(J e_{j}, e_{i}\right)-P\left(J e_{k}, e_{i}\right) \widetilde{g}\left(J e_{j}, e_{h}\right) \\
& +P\left(J e_{j}, e_{i}\right) \widetilde{g}\left(J e_{k}, e_{h}\right)-P\left(J e_{j}, e_{h}\right) \widetilde{g}\left(J e_{k}, e_{i}\right) \\
& -2\left\{P\left(J e_{k}, e_{j}\right) \widetilde{g}\left(J e_{i}, e_{h}\right)+P\left(J e_{i}, e_{h}\right) \widetilde{g}\left(J e_{k}, e_{j}\right)\right\}, \\
& 4\left\{\widetilde{g}\left(\sigma_{j a}, \sigma_{i h}\right)-\widetilde{g}\left(\sigma_{j h}, \sigma_{i a}\right)\right\}=3\left(P_{j a} \delta_{i h}-P_{i a} \delta_{j h}\right) \\
& -P\left(J e_{j}, e_{a}\right) \widetilde{g}\left(J e_{i}, e_{h}\right)+P\left(J e_{j}, e_{a}\right) \widetilde{g}\left(J e_{j}, e_{h}\right) \\
& +2 P\left(J e_{h}, e_{a}\right) \widetilde{g}\left(J e_{j}, e_{i}\right) \text {, } \\
& 2\left\{\widetilde{g}\left(\sigma_{i a}, \sigma_{h b}\right)-\widetilde{g}\left(\sigma_{i b}, \sigma_{h a}\right)\right\}=-c \widetilde{g}\left(J e_{i}, e_{h}\right) \widetilde{g}\left(T e_{b}, e_{a}\right) \\
& +P\left(J e_{b} . e_{a}\right) \widetilde{g}\left(J e_{i}, e_{h}\right)+P\left(J e_{i}, e_{h}\right) \widetilde{g}\left(T e_{b}, e_{a}\right) \text {, }
\end{aligned}
$$




$$
\begin{aligned}
& 4\left\{\widetilde{g}\left(\sigma_{i h}, \sigma_{b a}\right)-\widetilde{g}\left(\sigma_{i a}, \sigma_{b h}\right)\right\}=4 f_{1}^{2}\left\{\left(2+f_{1}^{2}\right)\left(e_{b} \log f_{1}\right)\left(e_{a} \log f_{1}\right)\right. \\
& \left.\quad+\nabla^{\mathcal{D}^{\theta}}{ }_{e_{b}} \nabla^{\mathcal{D}^{\theta}}{ }_{e_{a}} \log f_{1}\right\} \delta_{i h}+c\left\{\delta_{i h} \delta_{b a}+\widetilde{g}\left(J e_{i}, e_{h}\right) \widetilde{g}\left(T e_{b}, e_{a}\right)\right\} \\
& \quad 3\left(P_{i h} \delta_{b a}+P_{b a} \delta_{i h}\right)-P\left(J e_{i}, e_{h}\right) \widetilde{g}\left(T e_{b}, e_{a}\right) \\
& \quad-P\left(J e_{b}, e_{a}\right) \widetilde{g}\left(J e_{i}, e_{h}\right) \\
& 4\left\{\widetilde{g}\left(\sigma_{h a}, \sigma_{c b}\right)-\widetilde{g}\left(\sigma_{h b}, \sigma_{c a}\right)\right\}=3\left(P_{h a} \delta_{c b}-P_{h b} \delta_{c a}\right) \\
& \quad-P\left(J e_{h}, e_{a}\right) \widetilde{g}\left(T e_{c}, e_{b}\right)+P\left(J e_{h}, e_{b}\right) \widetilde{g}\left(T e_{c}, e_{a}\right) \\
& \quad+2 P\left(J e_{h}, e_{c}\right) \widetilde{g}\left(T e_{b}, e_{a}\right), \\
& 4\left\{\widetilde{g}\left(\sigma_{d a}, \sigma_{c b}\right)-\widetilde{g}\left(\sigma_{d b}, \sigma_{c h}\right)\right\}=-4 R^{\mathcal{D}^{\theta}} \\
& \quad+\widetilde{g}\left(T e_{d}, e_{a}\right) \widetilde{g}\left(T e_{c}, e_{b}\right)-\widetilde{g}\left(T e_{d}, e_{b}\right) \widetilde{g}\left(T e_{c}, e_{a}\right) \\
& \left.-2 \widetilde{g}\left(T e_{d}, e_{c}\right) \widetilde{g}\left(T e_{b}, e_{a}\right)\right\} \\
& +3\left(P \delta_{d a} \delta_{c b}-P \delta_{d b} \delta_{c a}+P_{c b} \delta_{d a}-P_{c a} \delta_{d b}\right) \\
& -P\left(J e_{d}, e_{a}\right) \widetilde{g}\left(T e_{c}, e_{b}\right)+P\left(J e_{d}, e_{b}\right) \widetilde{g}\left(T e_{c}, e_{a}\right) \\
& -P\left(J e_{c}, e_{b}\right) \widetilde{g}\left(T e_{d}, e_{a}\right)+P\left(J e_{c}, e_{a}\right) \widetilde{g}\left(T e_{d}, e_{b}\right) \\
& +2\left\{P\left(J e_{d}, e_{c}\right) \widetilde{g}\left(T e_{b}, e_{a}\right)+P\left(J e_{b}, e_{a}\right) \widetilde{g}\left(T e_{d}, e_{c}\right)\right\}
\end{aligned}
$$

for any

$$
k, j, i, h \in\{1,2, \ldots, 2 p\}
$$

and

$$
d, c, b, a \in\{2 p+1,2 p+2, \ldots, 2 p+q\},
$$

where we put $\sigma\left(e_{\mu}, e_{\lambda}\right)=\sigma_{\mu \lambda}$, etc.

The mean curvature vector $H$ and the mean curvature $\|H\|$ are respectively given by

$$
H=\frac{1}{n} \sum_{\mu=1}^{n} \sigma_{\mu \mu}, \quad\|H\|^{2}=\frac{1}{n^{2}} \sum_{\mu, \lambda=1}^{n} \widetilde{g}\left(\sigma_{\mu \mu}, \sigma_{\lambda \lambda}\right)
$$

and the length $\|\sigma\|$ of the second fundamental form $\sigma$ is given by

$$
\|\sigma\|^{2}=\sum_{\mu, \lambda=1}^{n} \widetilde{g}\left(\sigma_{\mu \lambda}, \sigma_{\mu \lambda}\right)=\sum_{\mu, \lambda=1}^{n} \sum_{r=n+1}^{m}\left\{\widetilde{g}\left(\sigma_{\mu \lambda}, e_{r}\right)\right\}^{2}
$$

for any local orthonormal frame $\left\{e_{1}, e_{2}, \ldots, e_{m}\right\}$ of $T \widetilde{M}$.

By virtue of the Gauss equations, we have

$$
\sum_{\mu, \lambda=1}^{n}\left(R_{\mu \lambda \mu \lambda}-\widetilde{R}_{\mu \lambda \mu \lambda}\right)=\|\sigma\|^{2}-n\|H\|^{2} .
$$


On the other hand, we have from (4.10) that

$$
\begin{aligned}
& 4 \sum_{\mu, \lambda=1}^{n} \widetilde{R}_{\mu \lambda \mu \lambda}=-\left(n^{2}+2 n-3 q\right) c-6(n-2) \sum_{\mu=1}^{n} P_{\mu \mu} \\
& \quad-6 \sum_{b=2 p+1}^{2 p+q} P_{b b}-3 c \sum_{b, a=2 p+1}^{2 p+q} T_{b a} T_{b a}+6 \sum_{b, a=2 p+1}^{2 p+q} P\left(J e_{b}, e_{a}\right) T_{b a},
\end{aligned}
$$

where $T_{b a}=\widetilde{g}\left(T_{b}{ }^{c} e_{c}, e_{a}\right)$ for any $c, b, a \in\{2 p+1,2 p+2, \ldots, 2 p+q=n\}$. We know $T_{b a}$ is skew-symmetric.

Moreover, we have from (1.9) and (4.7) that

$$
\begin{array}{r}
4 \sum_{\mu, \lambda=1}^{n} R_{\mu \lambda \mu \lambda}=-\left(e^{f_{1}^{2}} \tau^{\mathcal{D}}+\tau^{\mathcal{D}^{\theta}}\right)+8 p f_{1}^{2}\left\{(2 p-1) f_{1}^{2}\left\|\nabla^{\mathcal{D}^{\theta}} \log f_{1}\right\|^{2}+\right. \\
\left.\quad+2\left(2+f_{1}^{2}\right) \sum_{a=2 p+1}^{2 p+q}\left(e_{a} \log f_{1}\right)^{2}+2 \sum_{a=2 p+1}^{2 p+q} \nabla^{\mathcal{D}^{\theta}}{ }_{e_{a}} \nabla^{\mathcal{D}^{\theta}} e_{a} \log f_{1}\right\}
\end{array}
$$

where $\tau^{\mathcal{D}}$ (resp. $\tau^{\mathcal{D}^{\theta}}$ ) denotes the scalar curvature with respect to $g_{\mathcal{D}}$ (resp. $\left.g_{\mathcal{D}^{\theta}}\right)$.

Substituting (4.15) and (4.16) into (4.14), we obtain

$$
\begin{aligned}
4\|\sigma\|^{2} & =4 n\|H\|^{2}+8 p f\left\{(2 p-1) f_{1}^{2}\left\|\nabla^{\mathcal{D}^{\theta}} \log f_{1}\right\|^{2}+\right. \\
& \left.+2\left(2+f_{1}^{2}\right) \sum_{a=2 p+1}^{2 p+q}\left(e_{a} \log f_{1}\right)^{2}\right\}+\left(n^{2}+2 n-3 q\right) c+ \\
& +3 c \sum_{b, a=2 p+1}^{2 p+q}\left(T_{b a}\right)^{2}-4\left(e^{f_{1}^{2}} \tau^{\mathcal{D}}+\tau^{\mathcal{D}^{\theta}}\right)+ \\
& +16 p f_{1}^{2} \sum_{b, a=2 p+1}^{2 p+q} \nabla_{e_{a}}^{\mathcal{D}^{\theta}} \nabla_{e_{a}}^{\mathcal{D}^{\theta}} \log f_{1}+6(n-2) \sum_{\mu=1}^{n} P_{\mu \mu}+ \\
& +6 \sum_{a=2 p+1}^{2 p+q} P_{a a}-6 \sum_{b, a=2 p+1}^{2 p+q} P\left(J e_{b}, e_{a}\right) T_{b a} .
\end{aligned}
$$

Thus we have 
Theorem 4.1. In a first type warped product semi-slant submanifold in an l.c.K.-space form, the mean curvature satisfies the inequality

$$
\begin{aligned}
4 n\|H\|^{2} & +8 p f_{1}^{2}\left\{(2 p-1) f_{1}^{2}\left\|\nabla^{\mathcal{D}^{\theta}} \log f_{1}\right\|^{2}\right. \\
& \left.+2\left(2+f_{1}^{2}\right) \sum_{a=2 p+1}^{2 p+q}\left(e_{a} \log f_{1}\right)^{2}\right\}+\left(n^{2}+2 n-3 q\right) c \\
& +3 c \sum_{b, a=2 p+1}^{2 p+q}\left\{T_{b a}\right\}^{2}-4\left(e^{f_{1}^{2}} \tau^{\mathcal{D}}+\tau^{\mathcal{D}^{\theta}}\right) \\
& +16 p f_{1}^{2} \sum_{b, a=2 p+1}^{2 p+q} \nabla_{e_{a}}^{\mathcal{D}^{\theta}} \nabla_{e_{a}}^{\mathcal{D}^{\theta}} \log f_{1}+6(n-2) \sum_{\mu=1}^{n} P_{\mu \mu} \\
& +6 \sum_{a=2 p+1}^{2 p+q} P_{a a}-6 \sum_{b, a=2 p+1}^{2 p+q} P\left(J e_{b}, e_{a}\right) T_{b a} \geq 0 .
\end{aligned}
$$

Corollary 4.2. Under the same condition with Theorem 4.1, the equality case of (4.18) is that the submanifold is locally totally geodesic and the warping function $f_{1}$ satisfies

$$
\begin{aligned}
& 8 p f_{1}^{2}\left\{(2 p-1) f_{1}^{2}\left\|\nabla^{\mathcal{D}^{\theta}} \log f_{1}\right\|^{2}+\right. \\
& \left.\quad+2\left(2+f_{1}^{2}\right) \sum_{a=2 p+1}^{2 p+q}\left(e_{a} \log f_{1}\right)^{2}\right\}+\left(n^{2}+2 n-3 q\right) c+ \\
& \quad+3 c \sum_{b, a=2 p+1}^{2 p+q}\left\{T_{b a}\right\}^{2}-4\left(e^{f_{1}^{2}} \tau^{\mathcal{D}}+\tau^{\mathcal{D}^{\theta}}\right)+ \\
& \quad+16 p f_{1}^{2} \sum_{b, a=2 p+1}^{2 p+q} \nabla^{\mathcal{D}^{\theta}} e_{a} \nabla^{\mathcal{D}^{\theta}} e_{a} \log f_{1}+6(n-2) \sum_{\mu=1}^{n} P_{\mu \mu}+ \\
& \quad+6 \sum_{a=2 p+1}^{2 p+q} P_{a a}-6 \sum_{b, a=2 p+1}^{2 p+q} P\left(J e_{b}, e_{a}\right) T_{b a}=0 .
\end{aligned}
$$

and

$$
\begin{gathered}
\left(n^{2}+2 n-3 q\right) c+3 c \sum_{b, a=2 p+1}^{2 p+q}\left\{T_{b a}\right\}^{2}-4\left(e^{f_{1}^{2}} \tau^{\mathcal{D}}+\tau^{\mathcal{D}^{\theta}}\right)+ \\
16 p f_{1}^{2} \sum_{b, a=2 p+1}^{2 p+q} \nabla^{\mathcal{D}^{\theta}} e_{a} \nabla^{\mathcal{D}^{\theta}} e_{a} \log f_{1}+6(n-2) \sum_{\mu=1}^{n} P_{\mu \mu}+
\end{gathered}
$$




$$
+6 \sum_{a=2 p+1}^{2 p+q} P_{a a}-6 \sum_{b, a=2 p+1}^{2 p+q} P\left(J e_{b}, e_{a}\right) T_{b a} \leq 0 .
$$

\section{ACKNOWLEDGEMENTS}

The author is thankful to the referee for his/her valuable comments which have improved the manuscript.

\section{REREFENCES}

[1] Aurel Bejancu. CR submanifolds of a Kaehler manifold. I. Proc. Amer. Math. Soc., 69(1):135-142, 1978.

[2] Aurel Bejancu. CR submanifolds of a Kaehler manifold. II. Trans. Amer. Math. Soc., 250:333-345, 1979.

[3] Aurel Bejancu. Geometry of CR-submanifolds, volume 23 of Mathematics and its Applications (East European Series). D. Reidel Publishing Co., Dordrecht, 1986.

[4] Vittoria Bonanzinga, Koji Matsumoto. Semi-slant submanifolds in locally conformal Kaehler manifolds. to appear.

[5] Vittoria Bonanzinga, Koji Matsumoto. Warped product CR-submanifolds in locally conformal Kaehler manifolds. Period. Math. Hungar., 48(1-2):207-221, 2004.

[6] Bang-Yen Chen. Geometry of submanifolds. Marcel Dekker, Inc., New York, 1973. Pure and Applied Mathematics, No. 22.

[7] Bang-Yen Chen. CR-submanifolds of a Kaehler manifold. I. J. Differential Geom., 16(2):305-322, 1981.

[8] Bang-Yen Chen. CR-submanifolds of a Kaehler manifold. II. J. Differential Geom., 16(3):493-509 (1982), 1981.

[9] Bang-Yen Chen. Geometry of slant submanifolds. Katholieke Universiteit Leuven, Louvain, 1990.

[10] Toyoko Kashiwada. Some properties of locally conformal Kähler manifolds. Hokkaido Math. J., 8(2):191-198, 1979.

[11] Viqar Azam Khan, Meraj Ali Khan. Semi-slant submanifolds of a nearly Kaehler manifold. Turkish J. Math., 31(4):341-353, 2007.

[12] Barrett O'Neill. Semi-Riemannian geometry, volume 103 of Pure and Applied Mathematics. Academic Press, Inc. [Harcourt Brace Jovanovich, Publishers], New York, 1983. With applications to relativity.

[13] Neculai Papaghiuc. Semi-slant submanifolds of a Kaehlerian manifold. An. Ştiinţ. Univ. Al. I. Cuza Iaşi Secţ. I a Mat., 40(1):55-61, 1994.

[14] Izu Vaisman. On locally conformal almost Kähler manifolds. Israel Journal of Mathematics, 24(3-4):338-351, Dec 1976.

Received: July 3, 2017, accepted: September 2, 2017.

Koji Matsumoto

2-3-65 Nishi-Odori, Yonezawa, Yamagata, 992-0059, JAPAN

Email: tokiko_matsumoto@yahoo.com 